\title{
Roles of Exosomes and Exosomal MicroRNAs in Postoperative Sleep Disturbance
}

\author{
Xiangyi Gu \\ Junchao Zhu
}

Department of Anesthesiology, Shengjing Hospital of China Medical University, Shenyang, Liaoning, People's Republic of China
Correspondence: Junchao Zhu

Department of Anesthesiology, Shengjing

Hospital of China Medical University,

Shenyang, Liaoning, People's Republic of

China

Email zhujunchaol@hotmail.com

\begin{abstract}
Postoperative sleep disturbance (PSD) often occurs in elderly patients after major surgery and exerts harmful effects on postoperative recovery. PSD may increase the incidence of postoperative fatigue, severe anxiety and depression, pain sensitivity, and cognitive dysfunction, which can cause or aggravate neurodegenerative diseases via amyloid aggregation and tau accumulation. Exosomes are important carriers that mediate the transfer of active substances and genetic information among cells. Recent evidence has shown that exosomes are involved in the pathogenesis of end-organ morbidity caused by sleep disorders via increasing amyloid plaque formation, transmitting tau protein, regulating neuroinflammation, and increasing blood-brain barrier permeability. Additionally, exosomes may be useful for delivering therapeutic genetic materials, such as microRNAs (miRNAs) and proteins, to exert neuroprotective effects and reduce cognitive impairment. However, the molecular mechanisms underlying this process remain to be fully elucidated. This review focuses on exosome-related pathways and the modulatory role of exosomal miRNAs on the pathogenesis of sleep disturbance and neurodegeneration. Moreover, we discuss the advantages of reducing neurotoxic proteins via exosomal intervention and miRNA regulation. Future research in exosome administration may offer new insights into PSD-related pathomechanisms and therapeutics.
\end{abstract}

Keywords: postoperative sleep disturbance, exosomes, exosomal miRNAs

\section{Introduction}

Postoperative sleep disturbance (PSD) is a common complication after a major surgery, ${ }^{1,2}$ with patients often presenting with reduced sleep quality and duration, early awakening, frequent nightmares, sleep terrors, and sleep-related breathing disorders. ${ }^{3}$ Polysomnography studies on patients with sleep disorders have suggested occurrence of sleep fragmentation and deprivation as well as decrease in rapid eye movement (REM) and slow-wave sleep (SWS) after surgery. ${ }^{4}$ Additionally, PSD seriously affects the quality of life and increases the difficulty and burden of nursing care. The onset of sleep disorders is related to multiple factors, including age, preoperative complications, type of anaesthesia, degree of surgical trauma, and postoperative pain. Moreover, studies show that sleep disorders can be related to changes in postoperative brain function and increase the risk of postoperative cognitive decline. ${ }^{5,6}$ Early recognition and intervention of sleep disorders can reduce the incidence of mental dysfunction and cardiovascular events, improve prognosis, and shorten the duration of hospital stay. ${ }^{7}$

Exosomes are extracellular vesicles (diameter: 30-100 nm) that contain various active substances, such as proteins, small-interfering (si)RNAs, and microRNAs 
(miRNAs), and act as important carriers that mediate information exchange and material transfer among cells. ${ }^{8}$ Regulatory molecules, such as miRNAs, are transported to recipient cells via exosomes, where they affect biological pathways, leading to changes in cell function and pathology. ${ }^{9}$ Several recent studies have shown that nerve cellderived exosomes are related to pathological protein aggregation, synaptic morphology, and neuroinflammation, and that changes in exosome biological characteristics and level of dysfunction might be involved in neurodegeneration. Goetzl et $\mathrm{al}^{10,11}$ reported that in Alzheimer's disease (AD), P-S396tau, P-T181-tau, and A $\beta 1-42$ are elevated in plasma nerve cell-derived exosomes and can be used to predict the transition from preclinical $\mathrm{AD}$ to $\mathrm{AD}$. This review summarised current perspectives regarding the role of exosomes and exosomal miRNAs in PSD pathogenesis. The passways and pathomechanisms of relevant miRNAs is shown in Table 1.

\section{Postoperative Sleep Disturbance Sleep Structure and Function}

The normal sleep-wake cycle is naturally rhythmic. ${ }^{12}$ According to the American Academy of Sleep Medicine, sleep-wake states can be divided into wakefulness, nonREM sleep, and REM sleep. ${ }^{13}$ Compared with NREM, REM sleep is associated with stronger physiological activities, fluctuations in blood pressure and heart rate, irregular breathing, and increased brain metabolism. ${ }^{14,15}$

Long-term reductions in sleep time or complete sleep deprivation can cause cognitive deficits. Limiting sleep time to $4 \mathrm{~h}$ to $6 \mathrm{~h}$ per night can lead to neurobehavioral impairment upon waking, along with polysomnography results indicating prolonged REM latency and slight increase in SWS. ${ }^{16}$ Additionally, sleep deprivation in the time window before and after learning can impair memory and learning ability, as REM sleep deprivation 3-6 h after learning significantly increases levels of brain-derived neurotrophic factor (BDNF) in the CA1 region of the hippocampus. ${ }^{17}$ Based on the effectiveness of anti-tumour necrosis factor (TNF)- $\alpha$ therapy, Sochal et al $^{18}$ evaluated serum BDNF levels in Crohn's disease patients and healthy controls to assess the relationship between BDNF concentration and the severity of insomnia, and identified a positive correlation between serum BDNF level and results of the Athens Insomnia Scale. These findings suggest that BDNF is involved in PSD-related mechanisms via modulating chronic inflammation.
The interstitial fluid (ISF) of the brain transports soluble proteins, metabolic waste, and excess extracellular fluid along the paravenous drainage pathway, which relies on aquaporin 4 expressed in glial cells. Approximately $55 \%$ of soluble $A \beta$ in the interstitium is cleared by the lymphoid pathway. ${ }^{19}$ During sleep, the interstitial volume of the cerebral cortex increases by up to $60 \%$, and the convection of ISF and cerebrospinal fluid (CSF) increases to enable effective clearance of $A \beta$. Both sleep and anaesthesia can improve clearance efficiency, indicating that the sleep state itself may be the main factor promoting the clearance of metabolic waste. ${ }^{20}$

\section{Effects of PSD on Postoperative Outcomes}

Patients often show decreased daytime physical strength, functional limitations, and vulnerable emotions, which negatively affect post-surgery recovery. Severe PSD is manifested by changes in the sleep cycle. REM is significantly reduced on the night after surgery, followed by a rebound of REM sleep in the following 2 to 4 days and an increase in the proportion of REM sleep in total sleep time. ${ }^{21}$ REM sleep rebound causes hemodynamic instability and changes in pulmonary ventilation, thereby increasing the incidence of postoperative fatigue, severe anxiety and depression, pain sensitivity, and cognitive dysfunction and also resulting in longer hospital stays. $^{22,23}$ Kessler et $\mathrm{al}^{24}$ found that sleep disorders and low daytime physical activity reflect delayed recovery after discharge. Therefore, sleep intervention may be important for timely rehabilitation. Moreover, improving the sleep environment is recommended to alleviate sleep disturbances in postoperative patients. ${ }^{25}$ Pharmacological methods, including administration of zolpidem, melatonin, and/or dexmedetomidine, have recently been employed to improve sleep post-discharge. Specifically, administration of zolpidem one night before and on the first night after surgery improves sleep quality and fatigue. ${ }^{3,26}$

Sleep disturbances in AD patients are characterised by increased duration of awakenings, loss of SWS and REM sleep, and excessive daytime napping. AD patients spend a significant proportion of their day asleep, although this almost completely comprises stages 1 and 2 sleep, which barely compensates for night-time absences of SWS and REM sleep. ${ }^{27,28}$ Thus, abnormal shifts in the sleep-wake rhythm of AD patients may be implicated in PSD, resulting in more prominent cognitive deficits. ${ }^{29}$ 


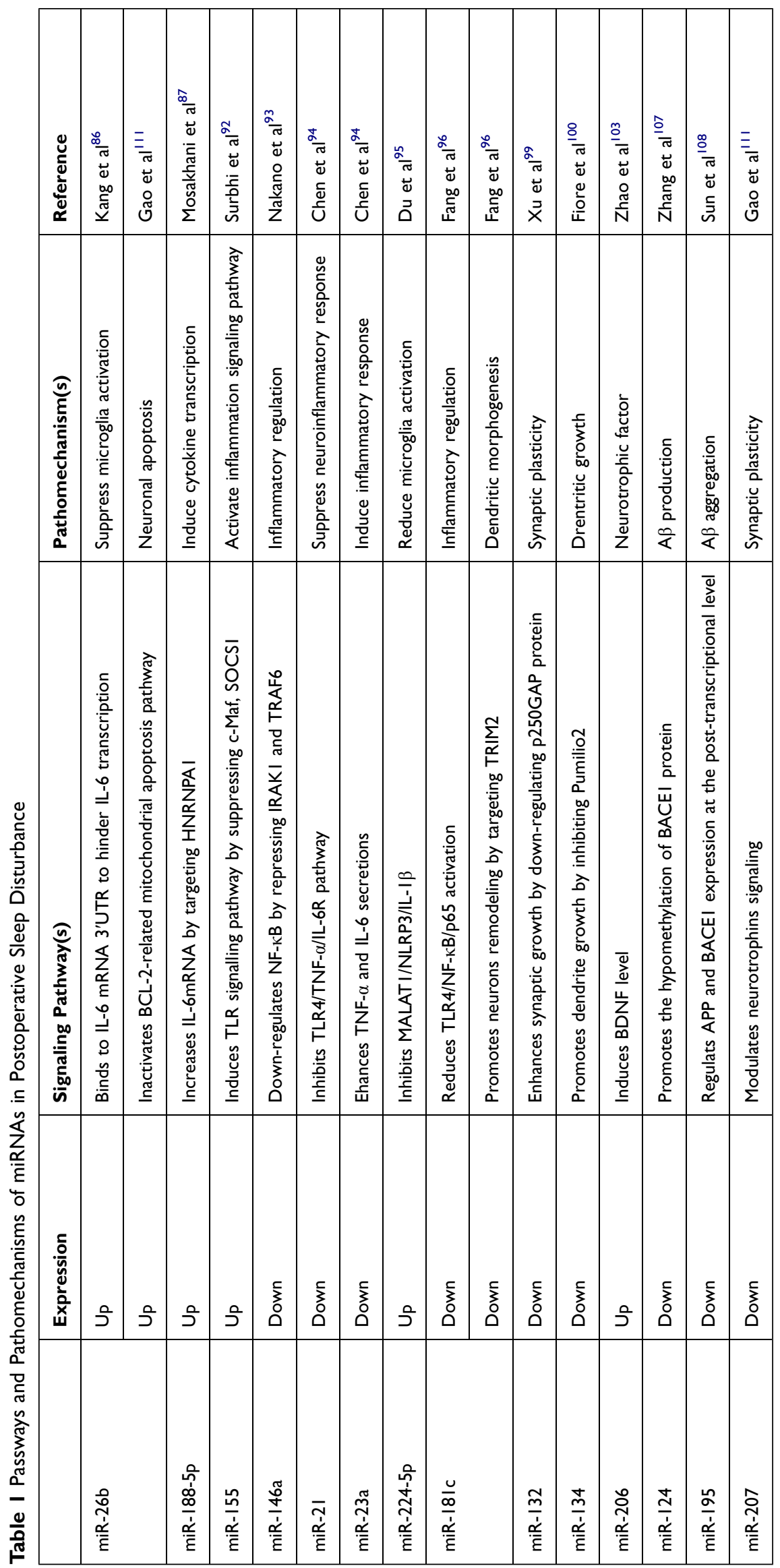




\section{Exosomes}

\section{Exosome Formation and Production}

Exosome formation begins in the endocytic system of the cell, with formation of intraluminal vesicles when the cell compartment is recessed inward and buds outward. Although exosomes were originally considered the "garbage bin" of cells, studies have revealed that these vesicles are not specific products of reticulocytes but are rather released from most mammalian cells. ${ }^{30}$ Later studies reported that exosomes released from mast cells contain $\sim 1200$ functional mRNAs, which could be transferred to other cells. ${ }^{31}$ Exosomes participate in several key biological processes by delivering biologically active substances, such as DNA, RNA (mRNAs, miRNAs, and circular RNAs), and proteins. In the CNS, neuronal cells, microglia, and astrocytes secrete exosomes. As mediators of cell-cell communication, exosomes are related to amyloid degradation, brain clearance, and intercellular diffusion of tau, which can induce neuronal apoptosis and lead to neuron loss. ${ }^{32}$

Exosome production varies according to different cell states. Dutkowska et $\mathrm{al}^{33}$ evaluated the expression of interleukin (IL)-1 $\beta$, IL-6, and IL-17 in tumours versus surgical margins, and observed significantly higher expression of IL-6 in tumours relative to adjacent tissue, thereby indicating that inflammatory processes play a role in tumorigenesis. Additionally, they evaluated miR-9 and miR-122 as cytokine regulators in pre- and postoperative peripheral blood exosomes, revealing elevated levels of both miRs after tumour resection.

A previous study investigating the expression of tissue inhibitor of metalloproteinases (TIMPs) in tumour and normal neighbouring tissues revealed decreased TIMP3 levels in non-cancerous tissue, whereas preoperative miR-17 expression in serum exosomes was significantly higher in cancer patients compared to healthy controls. ${ }^{34}$ These findings suggest that exosome production can be influenced by removal of tumour tissue, which could enable the detection of early biomarkers related to tumorigenesis and metastasis. ${ }^{35-37}$

\section{Exosomes and Sleep Disorders}

Rhythmic activities, such as the sleep-wake cycle, are regulated by the circadian rhythm, which is an autonomous, endogenous oscillator in all living organisms and comprises transcriptionally active clock genes (CLOCK, BMAL1, PER1, PER2, PER3, CRY1, and CRY2) and their protein products. Khalyfa et $\mathrm{al}^{38}$ found that Bmall, Cry2, and Perl expression was significantly reduced in plasma exosomes obtained from mice with sleep-rhythm disorders, indicating that exosomes can act as a bridge between peripheral clock-controlled genes and central rhythms and transmit the effects of circadian-rhythm disorders to target organs, thereby disturbing end-organ homeostasis. In type 2 diabetes, visceral white adipose tissue secretes exosomes that transport functional proteins and RNAs, resulting in alteration of metabolic functions in nearby and distant tissues. ${ }^{39}$ The associated molecular mechanism may involve $\alpha$-subunit of hypoxia-inducible factor 1 (HIF-1 $\alpha$ ), which regulates oxygen metabolism. Hepatocyte-specific Hifla knockout hindered this metabolic disorder by reducing GLP-1 degradation. ${ }^{40}$ Moreover, several studies investigating the impact of blood oxygen saturation on HIF-1 $\alpha$ mRNA levels showed decreases in these levels following continuous positive airway pressure (CPAP) treatment. ${ }^{41-43}$ These findings support the hypothesis that hypoxia is an independent risk factor for insulin resistance.

\section{The Role of Exosomes in the Pathogenesis of Sleep Disturbance Amyloid Aggregation}

Rajendran et $\mathrm{al}^{44}$ found that a fraction of $\mathrm{A} \beta$ in MVBs is loaded into exosomes and secreted into the extracellular environment. $A \beta$ and its products are transferred to MVBs, followed by APP hydrolysis by $\beta$-secretase, which mainly occurs in early endosomes. The association between exosomes and $A \beta$ suggests that inhibition of exosome secretion may reduce $\mathrm{AD}$-like pathological processes; however, exosome function in the process of $\mathrm{AD}$ remains controversial. ${ }^{45}$ Exosomes secreted in vivo in brains of APP-overexpressing mice contain higher levels of APP C-terminal fragments (CTFs) compared with normal brain tissue. These data support the hypothesis that an exosome-secretory pathway is beneficial to APP CTFs clearance. ${ }^{46} \mathrm{An}$ in vitro study by Kyongman et $\mathrm{al}^{47}$ found that exosomes derived from $\mathrm{N} 2 \mathrm{a}$ cells counteracted $\mathrm{A} \beta$-mediated damage to synaptic plasticity and rescued long-term potentiation. Exosomes enhance the uptake of $\mathrm{A} \beta$ into microglia through their surface glycosphingolipids, and ultimately reduce the formation of amyloid plaques.

The effect of sleep in amyloid pathogenesis has been assessed by wakefulness and sleep deprivation studies. Kang et $\mathrm{al}^{48}$ monitored hippocampal $\mathrm{A} \beta$ levels in vivo 
using microdialysis in both wild-type mice and human APP transgenic (Tg2576) mice. They found that A $\beta$ levels were significantly elevated during the waking period relative to the sleep period in control and Tg2576 mice, and that ISF $A \beta$ levels positively correlated with wake-time duration. Xie et $\mathrm{al}^{20}$ reported that sleep facilitates the clearance of $A \beta$ from the ISF via the glymphatic system as observed on the two-photon imaging of the brain of adult mice, which revealed improved exchange between the glymphatic system and the systemic circulation in the brains of sleeping or anesthetised mice relative to awake mice. These findings suggest that attenuated clearance of $A \beta$ under sleep-deprivation conditions may also contribute to increased ISF $A \beta$ levels.

Hypoxia can enhance $A \beta$ production via increased expression of $B A C E 1$, which increases the activity of $\beta$ secretase and increases APP hydrolysis. ${ }^{49} \mathrm{Bu}$ et $\mathrm{al}^{50}$ compared blood $A \beta$ levels under oxygen saturation in 49 patients with OSA, and found that plasma $A \beta$ levels positively correlated with hypoxic intensity. Additionally, to determine mechanism underlying hypoxia-induced increases in $\mathrm{A} \beta$, Xie et $\mathrm{al}^{51}$ investigated the effect of hypoxia on $\mathrm{A} \beta$ metabolism in a human neuroblastoma model stably expressing $A P P$. They found that $\mathrm{A} \beta$ export via exosomes increased after exposure to hypoxia, and that expression of CD147, a transmembrane glycoprotein present on exosomes, was also elevated under hypoxic conditions. CD147 is a subunit of $\gamma$-secretase, and its degradation is inhibited by the tubulin HOOK $1 .{ }^{52}$ Cui et $\mathrm{al}^{53}$ collected bone marrow-derived mesenchymal stem cells (MSCs) from App transgenic mice, found $\mathrm{A} \beta$ concentration decreased in the frontal cortex and hippocampus, and memory was improved which possibly due to an exosome-mediated increase in the expression of synapsin 1 and PSD95. Further clarification of the role of exosomes under hypoxic conditions will be beneficial for the prevention and treatment of abnormally increased $A \beta$ levels caused by sleep disorders.

\section{Tau Release and Transmission}

Sleep disorders cause hyperphosphorylation and aggregation of tau protein, resulting in formation of neurofibrillary tangles, neuritic plaques, and other structures. Compared with $A \beta$, pathological tau accumulation is more closely related to cognitive decline. ${ }^{54}$ Tau spreads in the brain in a layered manner by first aggregating in the entorhinal cortex and then spreading to the hippocampus before eventually extending to the neocortex and surrounding areas. Sleep regulates the metabolic homeostasis in neurons. Holth et $\mathrm{al}^{55}$ evaluated the effect of the sleep-wake cycle on tau levels in brain-tissue fluids in mice, and found that tau levels increased during the waking state and decreased during sleep; however not all protein levels increased in the CSF after sleep deprivation, and ISF tau level increased following prolonged wakefulness.

The mechanism associated with tau release and diffusion in the brain has long been a focus of tau-pathology research. Recent studies have suggested that tau protein may spread among neurons via exosomes. Wang et $\mathrm{al}^{56}$ found that cultured cerebral cortical neuron cells release tau through exosomes. However, compared with cytoplasmic tau, tau in exosomes was in a relatively lowphosphorylation state and accounted for $<2 \%$ of the total tau level. When intersynaptic connections are broken, exosomes cannot be taken up by neurons, which suggests that tau diffusion via exosomes depends on synaptic-structure integrity. Proteins (annexin 7 and Alix) extracted from the lysates of exosomes that secrete tau are involved in signal transduction and vesicle transport. ${ }^{57}$ The recruitment of these proteins to exosomes may promote tau release; however, the specific molecular mechanism associated with tau release via exosomes remains unknown, and further research is needed to confirm this hypothesis.

In vitro experiments indicate that tau mutations enhance their phosphorylation to promote tau release. ${ }^{58}$ Recent evidence shows that sleep deprivation can activate different kinases and phosphatases in the brain, leading to tau hyperphosphorylation. ${ }^{59}$ However, due to the dephosphorylation of tau by non-specific alkaline phosphatase in the brain, both phosphorylated and dephosphorylated tau proteins can exist outside the cells; therefore, it remains unclear whether phosphorylation promotes tau release.

\section{Blood-Brain Barrier (BBB) Integrity}

The BBB is a multicellular vascular structure comprising pericytes, astrocytes, end feet, brain endothelial cells, and the tight junctions between endothelial cells, which control the metabolic exchange between brain and peripheral circulation to protect brain tissue from microorganisms or toxins. ${ }^{60}$ Normal BBB function maintains brain homeostasis. Certain molecules in the brain, such as amyloid protein. TNF- $\alpha$, and prostaglandins, move in and out of the CNS rhythmically. ${ }^{61} \mathrm{~A} \beta$ periodically oscillates in the ISF of mouse brain tissues, and circadian-rhythm disorders can affect $A \beta$ metabolism. Lack of the circadian-clock gene BMAL1 leads to a decrease in daily $\mathrm{A} \beta$ oscillations 
and an increase in amyloid plaque formation. ${ }^{62}$ The transport of $A \beta$ through the BBB is one of the main steps in the $\mathrm{A} \beta$ metabolism. In rodents, ISF enters the brain parenchyma from the paravascular space of the arteries and flows out from the venous vascular space via the glymphatic system. Sleep can promote this pathway for clearing metabolic waste from the brain, and the interstitial space can increase by up to $60 \%$ during sleep, which may promote ISF and CSF convection. ${ }^{20}$

Decreased clearance of $A \beta$ through the BBB is one of the mechanisms of pathological $\mathrm{A} \beta$ deposition. ${ }^{63}$ Transporters at the BBB, such as receptor for advanced glycation end-products, low-density lipoprotein receptorrelated protein 1, apolipoprotein, and P-glycoprotein (P-gp), mediate $\mathrm{A} \beta$ transfer from the brain to peripheral circulation. ${ }^{64,65}$ Due to their structural and physiological characteristics, exosomes can enter the brain through the BBB. Pan et $\mathrm{al}^{66}$ used recombinant brain microvascular endothelial cell exosomes to increase the expression of P-gp receptors, and found that exosomes enter cells via endocytosis and prevent the lysosome-mediated degradation of P-gp receptors. In mice, increased intracellular $\mathrm{P}$-gp receptor levels reduce $\mathrm{A} \beta$ in the hippocampus and improve cognitive impairment caused by $A \beta$ aggregation.

After 6 days of sleep restriction, levels of the tightjunction proteins occludin, claudin-1, claudin-5, and zonula occludens (ZO)-2 were significantly reduced in the brain microvessels of mice but returned to the baseline levels in $24 \mathrm{~h}$ after resuming sleep. ${ }^{67}$ Khalyfa et $\mathrm{al}^{68}$ investigated the effects of plasma exosomes on different types of endothelial cells in 30 children with OSA. Compared with exosomes from children with normal cognitive function, plasma exosomes obtained from children with OSA altered the morphology of ZO-1 and increased BBB permeability, which indirectly affected the microenvironment and neural network in the brain. Following treatment with exosomes transfected with a specific mimic of miR-630, ZO-1 levels increased in endothelial cells, whereas transfection with selective inhibitors of miRNA-630 disrupted tight-junction permeability in endothelial cells. ${ }^{69}$

\section{Exosomal miRNAs}

\section{miRNAs in Neurodegeneration}

miRNAs are noncoding RNAs of $\sim 22$ nucleotides that exist widely in eukaryotes. It is estimated that the human genome encodes $>1000$ functional miRNAs that regulate the expression of $30 \%$ of protein-coding genes. ${ }^{70}$ In the nucleus, primary transcription products are hydrolysed by ribonuclease III Drosha to generate miRNA precursors. These precursors are transferred to the cytoplasm and processed by Dicer to form mature miRNAs that bind to the 3' untranslated region (UTR) of target mRNAs via RISC and Argonaute family proteins. This blocks translation initiation and results in translation inhibition or target degradation. ${ }^{71}$ As key regulators of neuronal morphology and function, miRNAs are overexpressed in the brain during different developmental stages of the CNS. Loss of Dicer can cause dopaminergic neuronal death and neurodegeneration, while loss of Dicer in the cortex and hippocampus affects nerve cell development, suggesting that miRNAs play important regulatory roles in various cellular processes, including neuronal morphogenesis, neuronal apoptosis, and neurodegeneration. ${ }^{72,73}$ With the discovery of exosome as a carrier of miRNAs, Xin et al demonstrated that in vitro MSCs stimulate the neurite outgrowth by transferring miR-133b to astrocytes and neurons via exosomes. ${ }^{74}$ The regulatory role of miRNAs has also been noted in the inflammatory response. In animal models of persistent pulmonary hypertension, exosomes mediate a decrease in levels of monocyte chemoattractant proteins and mitogens through miRNAs, thereby inhibiting macrophage infiltration and proinflammatorymediator release. $^{75}$

Mutations in the apolipoprotein gene are risk factors for $\mathrm{AD}$. The $\varepsilon 4$ allele of this gene is a genetic risk factor for protein-related pathology characterised by misfolded protein deposition in neurodegenerative diseases. ${ }^{76}$ Several studies have investigated the role of epigenetic mechanisms (mainly posttranscriptional modifications) in the pathogenesis of neurodegenerative diseases. Specifically, studies compared differential miRNA expression in the AD brain and revealed that miRNA-expression levels in the hippocampus, prefrontal cortex, CSF, and other tissues vary during the course of $\mathrm{AD} .{ }^{77}$ Neurodegeneration may result from changes in multiple cellular pathways. For example, miRNAs modulating central components of the amyloid cascade, such as APP and BACE1, have been identified. ${ }^{78,79}$ Moreover, in the CNS, neurons, microglia, and astrocytes can be regulated by miRNAs via exosomes. Immunomodulatory miRNAs are involved in activating the inflammatory response of microglia, which is reportedly a key pathomechanism in $\mathrm{AD}{ }^{80}$ Neurocognitive deficits are linked to loss of synaptic transmission and plasticity in murine models of $\mathrm{AD}$, as well 
as $\mathrm{AD}$ patients. In this review, we have focused on how various miRNAs exert their modulatory function on $\mathrm{AD}$ related biological pathways.

\section{Putative Roles of miRNAs in Sleep Disturbance and Pathogenesis} The Neuroinflammatory Response

After tissue injury or destruction, the inflammatory response can remove harmful substances and damaged tissues; however, excessive nervous system inflammation can cause neurotoxicity and cell death. ${ }^{81}$ Activation of microglia is an important step in initiating the neuroinflammatory response. Cytokines IL-1, IL-6, and TNF- $\alpha$ can induce microglial differentiation into the M1 (proinflammatory) phenotype, whereas IL-4, IL-10, and other cytokines promote their differentiation into the M2 (antiinflammatory) phenotype. ${ }^{82}$ Additionally, $\mathrm{A} \beta$ deposition, tau phosphorylation, and neurofibrillary tangles can induce microglial differentiation into the M1 phenotype, leading to impaired axonal transport and APP aggregation. ${ }^{83}$ Damaged neurons can also release $\mathrm{A} \beta$ to cause inflammation, initiating a cycle of continued $\mathrm{A} \beta$ release. CNS inflammation can affect neurotrophic factor levels, thereby damaging synaptic plasticity and leading to decreased nerve regeneration ability. ${ }^{84}$ Wadhwa et $\mathrm{al}^{85}$ showed that after $48 \mathrm{~h}$ sleep deprivation, levels of IL-1, IL-6, and TNF$\alpha$ increased significantly in the hippocampus of mice and resulted in impaired spatial memory; however, following minocycline treatment, the BDNF level increased significantly in the brain.

A previous report indicated that miR-26b suppressed microglial activation and decreased the levels of IL-6 in the CA1 region of the hippocampus. This study also showed that miR-26b can bind the $3^{\prime}$ UTR of IL-6 to inhibit its transcription, thereby effectively reducing neuronal apoptosis. ${ }^{86}$ Excessive daytime sleep leads to increased levels of miR-188-5p, which targets heterogeneous nuclear ribonucleoprotein A1 (HNRNPA1), a transcriptional regulator of IL-6. A previous study showed that HNRNPA1 overexpression increases IL-6 mRNA expression. ${ }^{87}$ miR-188-5p acts as a tumour suppressor and is related to ventricular remodelling and synaptic plasticity. ${ }^{88,89} \mathrm{Ni}$ et $\mathrm{al}^{90}$ reported that $B M A L 1$ expression in microglia is significantly reduced in $A p p$ transgenic mice with a disturbed sleep cycle, whereas mRNA expression of Tnfa, Illb, and $I l 6$ was increased. Experimental results indicate that decreased BMAL1 expression reduces the negative regulatory effect of $\mathrm{I} \kappa \mathrm{B} \alpha$ on $\mathrm{NF}-\kappa \mathrm{B}$, thus, upregulating $\mathrm{NF}-\kappa \mathrm{B}$ and leading to increased Tnfa, Illb, and Il6 transcription.

Toll-like receptors (TLRs) are surface receptors expressed on various cells, including microglia. TLR activation induces. Although TLRs are not exclusively expressed in microglia, the activation of downstream inflammatory signalling pathways by TLRs is an important step in AD pathogenesis. ${ }^{91}$ miRNAs can participate in TLR-mediated inflammation signalling pathways at different levels. miR-155 can promote TLR activation by inhibiting negative regulators of TLR, such as c-Maf and suppressor of cytokine signalling 1. In animal models, miR-155 knockout reduces the sleep-promoting effect of endotoxin, increases wakefulness time, and reduces NREM, suggesting its role as a mediator of the regulatory effect of sleep on the immune response. ${ }^{92}$ By contrast, miR-146a acts as an anti-inflammatory regulator in the brain. Upon injection of bone marrow-derived MSC exosomes into the brains of AD mice, microglia differentiated into the M2 phenotype, and astrocytes began to take up exosomes, followed by miR-146-mediated reductions in levels of NF- $\mathrm{NB} .^{93}$ In addition to miR-155 and miR146a, miR-21, miR-23, miR-224-5p, and miR-181c regulate proteins involved in the TLR signalling pathway. ${ }^{94-96}$ TRIM2, a target of miR-181c, reduces the ubiquitination of nerve-fibre filaments (neurofilament light), and promotes neuronal remodelling in the hippocampus exposed to hypoxia. ${ }^{96}$ Although activation of the inflammatory pathway is clearly an important factor in the development of cognitive impairment, additional research is needed to identify a direct link.

\section{Neuroregeneration}

The upregulated transcription of miRNAs in specific brain regions is driven by neuronal activity. For instance, miR132 expression is transcriptionally stimulated by neural activity and regulated by the cAMP response elementbinding protein (CREB) signalling pathway. $A \beta$ downregulates BDNF levels in AD by inhibiting the transcription of CREB, a downstream target of BDNF, thereby resulting in early decreases in miR-132. ${ }^{97}$ Furthermore, deletion of miR-132 in App transgenic mice enhances amyloid plaque accumulation and tau protein phosphorylation. ${ }^{98}$ However, in AD-induced synaptic dysfunction, the effects of miRNA-mediated downstream gene dysregulation remains unknown. 
Recent studies have revealed that a lack of miR-132 may lead to a decreased number of dendrites, which is closely related to the loss of synaptic function and cognitive impairment. Following transfection of miR-132 into $\mathrm{AD}$ mice, expression of the synaptic proteins PSD95, synapsin-1, and p-synapsin increased significantly in the temporal cortex and memory deficit was partially restored. ${ }^{99}$ Changes in synaptic plasticity and imbalances in the regulatory mechanism are related to the destruction of neural circuits, especially of the complex neuronal network formed by dendritic spines. There exists a complex miRNA-transcription-feedback system in the brain. For example, miR-134 expression is strictly regulated by synaptic activity via the transcription factor MEF2; miR134 promotes dendritic growth by inhibiting Pumilio2. ${ }^{100}$ Additionally, (sirtuin 1) SIRT1 is an NAD+-dependent protein acylase that can reduce miR-134 expression via an inhibitor complex containing the transcription factor YY1. A decrease in SIRT1 activity leads to increase in miR-134 levels, resulting in downregulation of CREB and BDNF levels, and impairment of synaptic plasticity. ${ }^{101}$ Patients with AD exhibit increased miR-134 expression in CSF, and $\mathrm{AD}$ exosomes contain higher levels of miR134. ${ }^{102}$ Furthermore, miR-134 can modulate exosomal transport, which is involved in $\mathrm{AD}$ pathogenicity, and miR-206-knockdown exosomes increase BDNF expression and inhibit neuronal apoptosis following acute brain injury. ${ }^{103}$ These findings suggest the feasibility of exosomal miRNAs as therapeutic markers.

\section{Cerebral Hemodynamic Changes}

Chronic cerebral hypoperfusion and decreased glucose metabolism before cognitive decline are high-risk factors for $\mathrm{AD} .{ }^{104}$ Due to decreased lung ventilation, brain metabolism and cerebral blood flow are reduced during NREM sleep. ${ }^{15}$ Brayet et al monitored cerebral blood flow during REM sleep and found that hypoperfusion of the anterior cingulate gyrus under REM is related to functional defects, which may lead to the onset of AD. ${ }^{105}$ miRNAs regulate BACE1 and APP expression at the posttranscriptional level; numerous transcription factor-binding sites have been identified in the promoter regions of $B A C E 1$ and $A P P$ to complement these miRNAs, suggesting an important role of miRNAs in the pathogenesis of cerebral bloodflow deficiency. ${ }^{106}$

In vivo experiments have shown that hypoxia or $A \beta$ downregulates miR-124 expression in the hippocampus. The underlying mechanism might involve activation of the exchange protein activated by cAMP (EPAC)-Rap1 pathway. Downregulation of miR-124 promotes hypomethylation of BACE1, increases BACE1 levels in the hippocampus, and leads to increased $A \beta$ production. ${ }^{107}$ Unlike miR-124, miR-195 can reduce $A \beta$ production under chronic cerebral hypoperfusion and thereby protect neurons; this effect is possibly related to negative regulation of NF- $\mathrm{kB} .{ }^{105,108}$ As an imbalanced miRNA expression is related to neuronal damage caused by cerebral insufficiency, promoting normal miRNA function may thus, have a protective effect on cerebral blood flow.

\section{Apoptosis}

Hypoxia, insufficient energy, inflammatory responses, and $\mathrm{A} \beta$ and tau proteins increase the accumulation of ROS in nerve cells and cause mitochondrial DNA mutations. miRNAs and their downstream molecules can regulate apoptosis pathways, as downregulation of miR-26 inactivates the BCL-2-related mitochondrial apoptosis pathway and activates Bax to induce apoptosis of liver cancer cells. ${ }^{109}$ Circadian-rhythm disorders affect DNA repair, cell cycle, and cell apoptosis and are associated with neurodegenerative diseases and cancer. ${ }^{110}$ Gao et al ${ }^{111}$ found that intermittent hypoxia affects the expression of pro-apoptotic and anti-apoptotic proteins in the hippocampus of a mouse model of OSA. Additionally, they found that miR-26b expression in the hippocampus increased three-fold, whereas miR-207 expression remained low in response to intermittent hypoxia. Moreover, miR-207 may play a protective role by participating in autophagy, as a murine model of PD showed that miR-207 inhibited apoptosis of mesencephalonderived dopaminergic neuronal cells, suggesting that miR207 may be a potential therapeutic target of PD. ${ }^{112}$ Recently, some studies investigated the feasibility of modulated miR$125 \mathrm{~b} / \mathrm{p} 38$ mitogen-activated protein kinase (MAPK) signalling to induce varying levels of neurons apoptosis. One study showed that upregulating p38 MAPK via loss of miR125b expression could regulate the expression of apoptosis-specific proteins in SH-SY5Y cells. ${ }^{113}$ Furthermore, p38 MAPK was reported to play a role in cascade reactions involved in inflammation, oxidative stress, and $\mathrm{A} \beta$-mediated cell apoptosis. ${ }^{114}$

\section{Exosomes and Exosomal miRNAs as Potential Treatment options for PSD}

A large body of evidence indicates that sleep disorders may be involved in the pathogenesis of neurodegenerative diseases and increase the risk of dementia. ${ }^{115}$ Exosomal miRNAs 
participate in sleep-disorder pathogenicity via two mechanisms: 1) direct regulation of signal pathway components or 2) regulation of proteins involved in signal pathways or key enzymes. ${ }^{16,117}$ Exosomes can carry altered genetic material derived from diseased cell and appear in early or advanced disease stages. In App transgenic mice, rabies virus-modified MSC-derived exosomes significantly reduced soluble A $\beta 40$ and $\mathrm{A} \beta 42$ levels in the brain. Furthermore, following exosome treatment, expression of TNF- $\alpha$ and IL-1 $\beta$ and levels of glial fibrillary acidic protein decreased, whereas glial cell function was significantly improved. ${ }^{118}$ In mice, MSC-derived exosomes significantly increased miR-21 levels after hypoxic preconditioning, resulting in downregulation of signal transducer and activator of transcription 3 phosphorylation and inhibition of NF-kB activation, which reduced the neuroinflammatory response in the brain. ${ }^{94}$ Additionally, other studies reported that miR-21 levels are related to attenuated inflammatory responses, and that reduced miR-206 expression via exosome delivery upregulates BDNF/tropomyosin receptor kinase B/CREB signalling, which exerts a neuroprotective effect on subarachnoid haemorrhage. ${ }^{103}$ Exosomes are naturally produced by human cells. Compared with other carriers of gene therapy, exosomes are advantageous in terms of achieving therapeutic effect, lower immune rejection, and better targeting. ${ }^{119}$ The delivery of therapeutic RNAs to target cells via exosomes for correcting protein dysfunction is a potential therapeutic strategy for brain diseases characterised by genetic abnormalities. ${ }^{120}$ Notably, exosomal miRNAs can act on different target genes, and expression of one gene can be regulated by multiple miRNAs. This suggests that intervention based on targeting the activity and/or treatment of a single gene would have limited efficacy. Moreover, sequential gene damage caused by sleep disorders requires a multi-pronged therapeutic approach for improving memory and learning dysfunctions.

\section{Conclusion}

Exosomes represent potential tools to therapeutically target sleep-disorder pathogenesis. Studies show that exosomes can promote protein misfolding and hinder their successful translation. Furthermore, miRNAs transferred by exosomes modulate the neuroinflammatory cascade, $\mathrm{A} \beta$ generation, and neuronal apoptosis. The role of exosomes in pathological mechanisms related to neuronal damage offers insights into their potential roles as biomarkers of and therapeutic targets for sleep-induced neuronal dysfunction. Elucidating the underlying mechanisms will promote the establishment of sleep-disorder models, prediction of dementia risk, and devise gene-therapy strategies. Future work should focus on detailed investigation of changes in exosome status and exosomal miRNAs under different types of sleep disturbance for disease prevention and early-stage diagnosis.

\section{Ethical Approval}

Not applicable

\section{Informed Consent}

Not applicable

\section{Funding}

No funding was received for this research.

\section{Disclosure}

There are no competing interests to declare.

\section{References}

1. Gögenur I, Wildschiøtz G, Rosenberg J. Circadian distribution of sleep phases after major abdominal surgery. $\mathrm{Br} J$ Anaesth. 2008;100(1):45-49. doi:10.1093/bja/aem340

2. Rampes S, Katie M, Divecha YA, et al. Postoperative sleep disorders and their potential impacts on surgical outcomes. J Biomed Res. 2019;34(4):271-280. doi:10.7555/JBR.33.20190054

3. Sipilä RM, Kalso EA. Sleep well and recover faster with less pain-a narrative review on sleep in the perioperative period. J Clin Med. 2021;10(9):2000. doi:10.3390/jcm10092000

4. Chung F, Liao P, Yegneswaran B, Shapiro CM, Kang W. Postoperative changes in sleep-disordered breathing and sleep architecture in patients with obstructive sleep apnea. Anesthesiology. 2014;120 (2):287-298. doi:10.1097/ALN.0000000000000040

5. Shokri-Kojori E, Wang G-J, Wiers CE, et al. $\beta$-Amyloid accumulation in the human brain after one night of sleep deprivation. Proc Natl Acad Sci U S A. 2018;115(17):4483-4488. doi:10.1073/ pnas. 1721694115

6. Bah TM, Goodman J, Iliff JJ. Sleep as a therapeutic target in the aging brain. Neurotherapeutics. 2019;16(3):554-568. doi:10.1007/s13311-019-00769-6

7. Su X, Wang D-X. Improve postoperative sleep: what can we do? Curr Opin Anaesthesiol. 2018;31(1):83-88. doi:10.1097/ ACO.0000000000000538

8. Cai Z-Y, Xiao M, Quazi SH. Exosomes: a novel therapeutic target for Alzheimer's disease? Neural Regen Res. 2018;13(5):930-935. doi:10.4103/1673-5374.232490

9. Cheng L, Sharples RA, Scicluna BJ, Hill AF. Exosomes provide a protective and enriched source of miRNA for biomarker profiling compared to intracellular and cell-free blood. $J$ Extracell Vesicles. 2014;3. doi:10.3402/jev.v3.23743.

10. Goetzl EJ, Kapogiannis D, Schwartz JB, et al. Decreased synaptic proteins in neuronal exosomes of frontotemporal dementia and Alzheimer's disease. FASEB $\quad J . \quad$ 2016;30(12):4141-4148. doi:10.1096/fj.201600816R

11. Goetzl EJ, Boxer A, Schwartz JB, et al. Altered lysosomal proteins in neural derived plasma exosomes in preclinical Alzheimer disease. Neurology. 2015;85(1):40-47. doi:10.1212/ WNL.0000000000001702 
12. Szmyd B, Rogut M, Białasiewicz P, et al. The impact of glucocorticoids and statins on sleep quality. Sleep Med Rev. 2021;55:101380. doi:10.1016/j.smrv.2020.101380

13. Gabryelska A, Szmyd B, Maschauer EL, et al. Utility of measuring CSF hypocretin-1 level in patients with suspected narcolepsy. Sleep Med. 2020;71:48-51. doi:10.1016/j.sleep.2020.03.009

14. McNamara P, Johnson P, McLaren D, et al. REM and NREM sleep mentation. Int Rev Neurobiol. 2010;92:69-86. doi:10.1016/ S0074-7742(10)92004-7

15. Berry RB, Brooks R, Gamaldo C, et al. AASM Scoring Manual Updates for 2017 (Version 2.4). J Clin Sleep Med. 2017;13 (5):665-666. doi:10.5664/jcsm.6576

16. Van Dongen HPA, Maislin G, Mullington JM, Dinges DF. The cumulative cost of additional wakefulness: dose-response effects on neurobehavioral functions and sleep physiology from chronic sleep restriction and total sleep deprivation. Sleep. 2003;26 (2):117-126. doi:10.1093/sleep/26.2.117

17. Karabulut S, Bayramov KK, Bayramov R, et al. Effects of post-learning REM sleep deprivation on hippocampal plasticity-related genes and microRNA in mice. Behav Brain Res. 2019;361:7-13. doi:10.1016/j.bbr.2018.12.045

18. Sochal M, Małecka-Panas E, Gabryelska A, et al. Brain-derived neurotrophic factor is elevated in the blood serum of Crohn's disease patients, but is not influenced by anti-TNF- $\alpha$ treatment-a pilot study. Neurogastroenterol Motil. 2020;e13978. doi:10.1111/ nmo. 13978

19. Iliff JJ, Wang M, Liao Y, et al. A paravascular pathway facilitates CSF flow through the brain parenchyma and the clearance of interstitial solutes, including amyloid $\beta$. Sci Transl Med. 2012;4 (147):147ra111. doi:10.1126/scitranslmed.3003748

20. Xie L, Kang H, Qiwu X, et al. Sleep drives metabolite clearance from the adult brain. Science. 2013;342(6156):373-377. doi:10.1126/science. 1241224

21. Chung F, Liao P, Elsaid H, et al. Factors associated with postoperative exacerbation of sleep-disordered breathing. Anesthesiology. 2014;120 (2):299-311. doi:10.1097/ALN.0000000000000041

22. Dette F, Cassel W, Urban F, et al. Occurrence of rapid eye movement sleep deprivation after surgery under regional anesthesia. Anesth Analg. 2013;116(4):939-943. doi:10.1213/ ANE.0b013e3182860e58

23. Kjølhede $P$, Langström $P$, Nilsson $P$, et al. The impact of quality of sleep on recovery from fast-track abdominal hysterectomy. J Clin Sleep Med. 2012;8(4):395-402. doi:10.5664/jcsm.2032

24. Kessler R, Knutson KL, Mokhlesi B, et al. Sleep and activity patterns in older patients discharged from the hospital. Sleep. 2019;42(11):zsz153. doi:10.1093/sleep/zsz153

25. Alessi CA, Martin JL, Webber AP, et al. Randomized, controlled trial of a nonpharmacological intervention to improve abnormal sleep/wake patterns in nursing home residents. J Am Geriatr Soc. 2005;53(5):803-810. doi:10.1111/j.1532-5415.2005.53251.x

26. Krenk L, Jennum P, Kehlet H. Postoperative sleep disturbances after zolpidem treatment in fast-track hip and knee replacement. J Clin Sleep Med. 2014;10(3):321-326. doi:10.5664/jcsm.3540

27. Musiek ES. Circadian clock disruption in neurodegenerative diseases: cause and effect? Front Pharmacol. 2015;6:29. doi: 10.3389/fphar.2015.00029

28. Vecchierin M-F. Sleep disturbances in Alzheimer's disease and other dementias. Psychol Neuropsychiatr Vieil. 2010;8(1):15-23. doi:10.1684/pnv.2010.0203

29. Henry A, Katsoulis M, Masi S, et al. The relationship between sleep duration, cognition and dementia: a Mendelian randomization study. Int J Epidemiol. 2019;48(3):849-860. doi:10.1093/ije/ dyz071

30. Raposo G, Stoorvogel W. Extracellular vesicles: exosomes, microvesicles, and friends. J Cell Biol. 2013;200(4):37383. doi:10.1083/jcb.201211138
31. Valadi H, Ekström K, Bossios A, et al. Exosome-mediated transfer of mRNAs and microRNAs is a novel mechanism of genetic exchange between cells. Nat Cell Biol. 2007;9(6):654-659. doi:10.1038/ncb1596

32. Kalluri R, LeBleu VS. The biology, function, and biomedical applications of exosomes. Science. 2020;367(6478):eaau6977. doi:10.1126/science.aau6977

33. Dutkowska A, Szmyd B, Kaszkowiak M, et al. Expression of inflammatory interleukins and selected miRNAs in non-small cell lung cancer. Sci Rep. 2021;11(1):5092. doi:10.1038/s41598-021-84408-1

34. Czarneck KH, Szmyd B, Barańska M, et al. A strong decrease in TIMP3 expression mediated by the presence of miR-17 and 20a enables extracellular matrix remodeling in the NSCLC lesion surroundings. Front Oncol. 2019;9:1372. doi:10.3389/ fonc.2019.01372

35. Zhang L, Shan X, Wang J, et al. A three-microRNA signature for lung squamous cell carcinoma diagnosis in Chinese male patients. Oncotarget. 2017;8(49):86897-86907. doi:10.18632/ oncotarget. 19666

36. Molina-Pinelo S, Dolores Pastor M, Suarez R, et al. MicroRNA clusters: dysregulation in lung adenocarcinoma and COPD. Eur Respir J. 2014;43(6):1740-1749. doi:10.1183/09031936.00091513

37. Aushev VN, Zborovskaya IB, Laktionov KK, et al. Comparisons of microRNA patterns in plasma before and after tumor removal reveal new biomarkers of lung squamous cell carcinoma. PLoS One. 2013;8(10):e78649. doi:10.1371/journal.pone.0078649

38. Khalyfa A, Poroyko VA, Qiao Z, et al. Exosomes and metabolic function in mice exposed to alternating dark-light cycles mimicking night shift work schedules. Front Physiol. 2017;8:882. doi:10.3389/fphys.2017.00882

39. Lee M-J, Park D-H, Kang J-H. Exosomes as the source of biomarkers of metabolic diseases. Ann Pediatr Endocrinol Metab. 2016;21(3):119-125. doi:10.6065/apem.2016.21.3.119

40. Gabryelska A, Karuga FF, Szmyd B, Białasiewicz P. HIF-1 $\alpha$ as a mediator of insulin resistance, T2DM, and its complications: potential links with obstructive sleep apnea. Front Physiol. 2020;11:1035. doi:10.3389/fphys.2020.01035

41. Gabryelska A, Szmyd B, Szemraj J, et al. Patients with obstructive sleep apnea present with chronic upregulation of serum HIF$1 \alpha$ protein. $J$ Clin Sleep Med. 2020;16(10):1761-1768. doi: $10.5664 /$ jesm. 8682

42. Gabryelska A, Szmyd B, Panek M, et al. Serum hypoxiainducible factor- $1 \alpha$ protein level as a diagnostic marker of obstructive sleep apnea. Pol Arch Intern Med. 2020;130 (2):158-160. doi:10.20452/pamw.15104

43. Gabryelska A, Sochal M, Turkiewicz S, et al. Relationship between HIF-1 and circadian clock proteins in obstructive sleep apnea patients-preliminary study. J Clin Med. 2020;9(5):1599. doi:10.3390/jcm9051599

44. Rajendran L, Honsho M, Zahn TR, et al. Alzheimer's disease beta-amyloid peptides are released in association with exosomes. Proc Natl Acad Sci U S A. 2006;103(30):11172-11177. doi:10.1073/pnas.0603838103

45. Dinkins MB, Dasgupta S, Wang G, et al. Exosome reduction in vivo is associated with lower amyloid plaque load in the 5XFAD mouse model of Alzheimer's disease. Neurobiol Aging. 2014;35 (8):1792-1800. doi:10.1016/j.neurobiolaging.2014.02.012

46. Perez-Gonzalez R, Gauthier SA, et al. The exosome secretory pathway transports amyloid precursor protein carboxyl-terminal fragments from the cell into the brain extracellular space. $J$ Biol Chem. 2012;287(51):43108-43115. doi:10.1074/jbc. M112.404467

47. Kyongman A, Klyubin I, Kim Y, et al. Exosomes neutralize synaptic-plasticity-disrupting activity of $\mathrm{A} \beta$ assemblies in vivo. Mol Brain. 2013;6:47. doi:10.1186/1756-6606-6-47 
48. Kang J-E, Lim MM, Bateman RJ, et al. Amyloid-beta dynamics are regulated by orexin and the sleep-wake cycle. Science. 2009;326(5955):1005-1007. doi:10.1126/science.1180962

49. Salminen A, Kauppinen A, Kaarniranta K. Hypoxia/ischemia activate processing of Amyloid Precursor Protein: impact of vascular dysfunction in the pathogenesis of Alzheimer's disease. J Neurochem. 2017;140(4):536-549. doi:10.1111/jnc.13932

50. Bu X-L, Liu Y-H, Wang Q-H, et al. Serum amyloid-beta levels are increased in patients with obstructive sleep apnea syndrome. Sci Rep. 2015;5:13917. doi:10.1038/srep13917

51. Xie J-C, Ma X-Y, Liu X-H, et al. Hypoxia increases amyloid- $\beta$ level in exosomes by enhancing the interaction between CD147 and Hook1. Am J Transl Res. 2018;10(1):150-163.

52. Zhou S, Zhou H, Walian PJ, Jap BK. The discovery and role of CD147 as a subunit of gamma-secretase complex. Drug News Perspect. 2006;19(3):133-138. doi:10.1358/dnp.2006.19.3.985932

53. Cui G-H, Jing W, Mou F-F, et al. Exosomes derived from hypoxia-preconditioned mesenchymal stromal cells ameliorate cognitive decline by rescuing synaptic dysfunction and regulating inflammatory responses in APP/PS1 mice. FASEB J. 2018;32 (2):654-668. doi:10.1096/fj.201700600R

54. Brier MR, Gordon B, Friedrichsen $\mathrm{K}$, et al. Tau and A $\beta$ imaging, CSF measures, and cognition in Alzheimer's disease. Sci Transl Med. 2016;8(338):338ra66. doi:10.1126/scitranslmed.aaf2362

55. Holth JK, Fritschi SK, Wang C, et al. The sleep-wake cycle regulates brain interstitial fluid tau in mice and CSF tau in humans. Science. 2019;363(6429):880-884. doi:10.1126/science. aav2546

56. Wang Y, Balaji V, Kaniyappan S, et al. The release and trans-synaptic transmission of Tau via exosomes. Mol Neurodegener. 2017;12(1):5. doi:10.1186/s13024-016-0143-y

57. Saman S, Lee NCY, Inoyo I, et al. Proteins recruited to exosomes by tau overexpression implicate novel cellular mechanisms linking tau secretion with Alzheimer's disease. J Alzheimers Dis 2014;40(Suppl 1):S47-70. doi:10.3233/JAD-132135

58. Plouffe V, Mohamed N-V, Rivest-McGraw J, et al. Hyperphosphorylation and cleavage at D421 enhance tau secretion. PLoS One. 2012;7(5):e36873. doi:10.1371/journal. pone.0036873

59. Barthélemy NR, Liu H, William L, et al. Sleep deprivation affects tau phosphorylation in human cerebrospinal fluid. Ann Neurol. 2020;87(5):700-709. doi:10.1002/ana.25702

60. Obermeier B, Daneman R, Ransohoff RM. Development, maintenance and disruption of the blood-brain barrier. Nat Med. 2013;19(12):1584-1596. doi:10.1038/nm.3407

61. Kress GJ, Liao F, Dimitry J, et al. Regulation of amyloid-beta dynamics and pathology by the circadian clock. J Exp Med. 2018;215(4):1059-1068. doi:10.1084/jem.20172347

62. Scott Miners J, Schulz I, Love S. Differing associations between $\mathrm{A} \beta$ accumulation, hypoperfusion, blood-brain barrier dysfunction and loss of PDGFRB pericyte marker in the precuneus and parietal white matter in Alzheimer's disease. J Cereb Blood Flow Metab. 2018;38(1):103-115. doi:10.1177/ $0271678 X 17690761$

63. Wan W, Chen H, Yaming L. The potential mechanisms of A $\beta$-receptor for advanced glycation end-products interaction disrupting tight junctions of the blood-brain barrier in Alzheimer's disease. Int J Neurosci. 2014;124(2):75-81. doi:10.3109/00207454.2013.825258

64. Cai Z, Qiao P-F, Wan C-Q, et al. Role of blood-brain barrier in Alzheimer's Disease. J Alzheimers Dis. 2018;63(4):1223-1234. doi:10.3233/JAD-180098

65. Amanda B, Anika CP, Hartz MS, et al. New evidence for P-gpmediated export of Amyloid- $\beta$ PEPTIDES in molecular, bloodbrain barrier and neuronal models. Int J Mol Sci. 2020;22(1):246. doi:10.3390/ijms22010246
66. Pan J, Ruyi H, Huo Q, et al. Brain microvascular endothelial cell derived exosomes potently ameliorate cognitive dysfunction by enhancing the clearance of $A \beta$ through up-regulation of P-gp in mouse model of AD. Neurochem Res. 2020;45(9):2161-2172. doi:10.1007/s11064-020-03076-1

67. Junyun $\mathrm{H}$, Hsuchou $\mathrm{H}, \mathrm{He} \mathrm{Y}$, et al. Sleep restriction impairs blood-brain barrier function. $J$ Neurosci. 2014;34 (44):14697-14706. doi:10.1523/JNEUROSCI.2111-14.2014

68. Khalyfa A, Gozal D, Kheirandish-Gozal. L. Plasma extracellular vesicles in children with OSA disrupt blood-brain barrier integrity and endothelial cell wound healing in vitro. Int $J$ Mol Sci. 2019;20(24):6233. doi:10.3390/ijms20246233

69. Khalyfa A, Kheirandish-Gozal L, Khalyfa AA, et al. Circulating plasma extracellular microvesicle MicroRNA cargo and endothelial dysfunction in children with obstructive sleep apnea. Am J Respir Crit Care Med. 2016;194(9):1116-1126. doi:10.1164/ rccm.201602-0323OC

70. Saliminejad K, Reza H, Khorshid K, et al. An overview of microRNAs: biology, functions, therapeutics, and analysis methods. J Cell Physiol. 2019;234(5):5451-5465. doi:10.1002/jcp.27486

71. Treiber T, Treiber N, Meister G. Regulation of microRNA biogenesis and its crosstalk with other cellular pathways. Nat Rev Mol Cell Biol. 2019;20(1):5-20. doi:10.1038/s41580-018-0059-1

72. Kim J, Inoue K, Ishii J, et al. MicroRNA feedback circuit in midbrain dopamine neurons. Science. 2007;317 (5842):1220-1224. doi:10.1126/science.1140481

73. Davis TH, Cuellar TL, Koch SM, et al. Conditional loss of dicer disrupts cellular and tissue morphogenesis in the cortex and hippocampus. J Neurosci. 2008;28(17):4322-4330. doi:10.1523/ JNEUROSCI.4815-07.2008

74. Xin H, Wang F, Yanfeng L, et al. Secondary release of exosomes from astrocytes contributes to the increase in neural plasticity and improvement of functional recovery after stroke in rats treated with exosomes harvested from MicroRNA 133b-overexpressing multipotent mesenchymal stromal cells. Cell Transplant. 2017;26 (2):243-257. doi:10.3727/096368916X693031

75. Lee C, Alex Mitsialis S, Aslam M, et al. Exosomes mediate the cytoprotective action of mesenchymal stromal cells on hypoxia-induced pulmonary hypertension. Circulation. 2012;126 (22):2601-2611. doi:10.1161/CIRCULATIONAHA.112.114173

76. Rodriguez-Vieitez E, Nielsen HM. Associations between APOE variants, Tau and $\alpha$-Synuclein. Adv Exp Med Biol. 2019;1184:177-186. doi:10.1007/978-981-32-9358-8_15

77. Kumar S, Hemachandra Reddy P. Are circulating microRNAs peripheral biomarkers for Alzheimer's disease? Biochim Biophys Acta. 2016;1862(9):1617-1627. doi:10.1016/j.bbadis.2016.06.001

78. Samadian M, Gholipour M, Hajiesmaeili M, et al. The eminent role of microRNAs in the pathogenesis of Alzheimer's Disease. Front Aging Neurosci. 2021;13:641080. doi:10.3389/ fnagi.2021.641080

79. Sun L-H, Ban T, Liu C-D, et al. Activation of Cdk5/p25 and tau phosphorylation following chronic brain hypoperfusion in rats involves microRNA-195 down-regulation. $J$ Neurochem. 2015;134(6):1139-1151. doi:10.1111/jnc.13212

80. Brites D, Fernandes A. Neuroinflammation and depression: microglia activation, extracellular microvesicles and microRNA dysregulation. Front Cell Neurosci. 2015;9:476. doi:10.3389/ fncel.2015.00476

81. Tang Y, Le W. Differential roles of M1 and M2 microglia in neurodegenerative diseases. Mol Neurobiol. 2016;53 (2):1181-1194. doi:10.1007/s12035-014-9070-5

82. Ransohoff RM. A polarizing question: do M1 and M2 microglia exist? Nat Neurosci. 2016;19(8):987-991. doi:10.1038/nn.4338

83. Krstic D, Knuesel I. Deciphering the mechanism underlying late-onset Alzheimer disease. Nat Rev Neurol. 2013;9(1):25-34. doi:10.1038/nrneurol.2012.236 
84. Giacobbo BL, Doorduin J, Klein HC, et al. Brain-derived neurotrophic factor in brain disorders: focus on neuroinflammation. Mol Neurobiol. 2019;56(5):3295-3312. doi:10.1007/s12035-018-1283-6

85. Wadhwa M, Prabhakar A, et al. Inhibiting the microglia activation improves the spatial memory and adult neurogenesis in rat hippocampus during $48 \mathrm{~h}$ of sleep deprivation. J Neuroinflammation. 2017;14(1):222. doi:10.1186/s12974-017-0998-Z

86. Kang Y-C, Zhang L, Ying S, et al. MicroRNA-26b regulates the microglial inflammatory response in hypoxia/ischemia and affects the development of vascular cognitive impairment. Front Cell Neurosci. 2018;12:154. doi:10.3389/fncel.2018.00154

87. Mosakhani N, Sarhadi V, Panula P, et al. Narcolepsy patients' blood-based miRNA expression profiling: miRNAexpression differences with Pandemrix vaccination. Acta Neurol Scand. 2017;136(5):462-469. doi:10.1111/ane.12749

88. Mishra PK, Tyagi N, Kundu S, Tyagi SC. MicroRNAs are involved in homocysteine-induced cardiac remodeling. Cell Biochem Biophys. 2009;55(3):153-162. doi:10.1007/s12013-009-9063-6

89. Lee K, Kim J-H, Kwon O-B, et al. An activity-regulated microRNA, miR-188, controls dendritic plasticity and synaptic transmission by downregulating neuropilin-2. J Neurosci. 2012;32(16):5678-5687. doi:10.1523/JNEUROSCI.6471-11.2012

90. Ni Junjun, Zhou W, Meng J, et al. An impaired intrinsic microglial clocksystem induces neuroinflammatory alterations in the early stage of amyloid precursor protein knock-in mouse brain. $J$ Neuroinflammation. 2019;16(1):173. doi:10.1186/s12974-0191562-9

91. Rahmani F, Rezaei N. Therapeutic targeting of Toll-like receptors: a review of Toll-like receptors and their signaling pathways in psoriasis. Expert Rev Clin Immunol. 2016;12(12):1289-1298. doi:10.1080/1744666X.2016.1204232

92. Surbhi, Borniger JC, Russart KLG, et al. miR-155 deletion modulates lipopolysaccharide-induced sleep in female mice. Chronobiol Int. 2019;36(2):188-202. doi:10.1080/ 07420528.2018 .1525617

93. Nakano M, Kubota K, Kobayashi E, et al. Bone marrow-derived mesenchymal stem cells improve cognitive impairment in an Alzheimer's disease model by increasing the expression of microRNA-146a in hippocampus. Sci Rep. 2020;10(1):10772. doi:10.1038/s41598-020-67460-1

94. Chen Y-C, Hsu P-Y, Su M-C, et al. miR-21-5p under-expression in patients with obstructive sleep apnea modulates intermittent hypoxia with re-oxygenation-induced-cell apoptosis and cytotoxicity by targeting pro-inflammatory TNF- $\alpha$-TLR4 signaling. Int J Mol Sci. 2020;21(3):999. doi:10.3390/ijms21030999

95. Du P, Wang J, Han Y, Feng J. Blocking the LncRNA MALAT1/ miR-224-5p/NLRP3 axis inhibits the hippocampal inflammatory response in T2DM with OSA. Front Cell Neurosci. 2020;14:97. doi:10.3389/fncel.2020.00097

96. Fang C, Qian L, Min G, et al. MicroRNA-181c ameliorates cognitive impairment induced by chronic cerebral hypoperfusion in rats. Mol Neurobiol. 2017;54(10):8370-8385. doi:10.1007/ s12035-016-0268-6

97. Rosa E, Fahnestock M. CREB expression mediates amyloid $\beta$ induced basal BDNF downregulation. Neurobiol Aging. 2015;36 (8):2406-2413. doi:10.1016/j.neurobiolaging.2015.04.014

98. Salta E, De Strooper B. microRNA-132: a key noncoding RNA operating in the cellular phase of Alzheimer's disease. FASEB J. 2017;31(2):424-433. doi:10.1096/fj.201601308

99. Nan X, Ang-Di L, Li-Li J, et al. miR-132 regulates the expression of synaptic proteins in APP/PS1 transgenic mice through C1q. Eur J Histochem. 2019;63(2):3008. doi:10.4081/ejh.2019.3008

100. Fiore R, Khudayberdiev S, Christensen M, et al. Mef2-mediated transcription of the miR379-410 cluster regulates activitydependent dendritogenesis by fine-tuning Pumilio2 protein levels. EMBO J. 2009;28(6):697-710. doi:10.1038/emboj.2009.10
101. Yamakuchi M. MicroRNA regulation of SIRT1. Front Physiol. 2012;3:68. doi:10.3389/fphys.2012.00068

102. Riancho J, Luis J, Vázquez-Higuera, et al. MicroRNA profile in patients with Alzheimer's Disease: analysis of miR-9-5p and miR-598 in raw and exosome enriched cerebrospinal fluid samples. J Alzheimers Dis. 2017;57(2):483-491. doi:10.3233/JAD161179

103. Zhao H, Yunjun L, Chen L, et al. HucMSCs-derived miR-206knockdown exosomes contribute to neuroprotection in subarachnoid hemorrhage induced early brain injury by targeting BDNF. Neuroscience. 2019;417:11-23. doi:10.1016/j.neuroscience.2 019.07.051

104. Daulatzai MA. Cerebral hypoperfusion and glucose hypometabolism: key pathophysiological modulators promote neurodegeneration, cognitive impairment, and Alzheimer's disease. $J$ Neurosci Res. 2017;95(4):943-972. doi:10.1002/jnr.23777

105. Brayet P, Petit D, Baril A-A, et al. Brain perfusion during rapid eye-movement sleep successfully identifies amnestic mild cognitive impairment. Sleep Med. 2017;34:134-140. doi:10.1016/j. sleep.2017.01.025

106. Hampel H, Vassar R, De Strooper B. The $\beta$-Secretase BACE1 in Alzheimer's disease. Biol Psychiatry. 2021;89(8):745-756. doi:10.1016/j.biopsych.2020.02.001

107. Zhang X, Huang X, Fang C, et al. miR-124 regulates the expression of BACE1 in the hippocampus under chronic cerebral hypoperfusion. Mol Neurobiol. 2017;54(4):2498-2506. doi:10.1007/s12035-016-9845-y

108. Sun JA-H, Che H, Zhang R, et al. MicroRNA-195 protects against dementia induced by chronic brain hypoperfusion via its anti-amyloidogenic effect in rats. $J$ Neurosci. 2013;33 (9):3989-4001. doi:10.1523/JNEUROSCI.1997-12.2013

109. Wang Y, Sun B, Zhao X, et al. Twist1-related miR-26b-5p suppresses epithelial-mesenchymal transition, migration and invasion by targeting SMAD1 in hepatocellular carcinoma. Oncotarget. 2016;7(17):24383-24401. doi:10.18632/oncotarget.8328

110. Maiese K. Moving to the rhythm with Clock (Circadian) genes, autophagy, mTOR, and SIRT1 in degenerative disease and cancer. Curr. Neurovasc Res. 2017;14(3):299-304. doi:10.2174/ 1567202614666170718092010

111. Gao H, Han Z, Huang S, et al. Intermittent hypoxia caused cognitive dysfunction relate to miRNAs dysregulation in hippocampus. Behav Brain Res. 2017;335:80-87. doi:10.1016/j. bbr.2017.06.025

112. Li L, Chen H-Z, Chen -F-F, et al. Global microRNA expression profiling reveals differential expression of target genes in 6-hydroxydopamine-injured MN9D cells. Neuromolecular Med. 2013;15(3):593-604. doi:10.1007/s12017-013-8244-z

113. Ren H, Qiu W, Qiuyun L, et al. Potential contribution of microRNA-125b targeting p38MAPK to relieving intermittent hypoxia-induced dementia of rat models. $J$ Clin Neurosci. 2019;64:234-241. doi:10.1016/j.jocn.2019.03.002

114. Zhou Y, Wang Z-F, Wei L, et al. Protective effects of microRNA330 on amyloid $\beta$-protein production, oxidative stress, and mitochondrial dysfunction in Alzheimer's disease by targeting VAV1 via the MAPK signaling pathway. J Cell Biochem. 2018;119 (7):5437-5448. doi:10.1002/jcb.26700

115. Jinlei L, Ogrodnik M, Kolachalama VB, et al. Assessment of the mid-life demographic and lifestyle risk factors of dementia using data from the Framingham heart study offspring cohort. $J$ Alzheimers Dis. 2018;63(3):1119-1127. doi:10.3233/JAD170917

116. Dehghani R, Rahmani F, Rezaei N. MicroRNA in Alzheimer's disease revisited: implications for major neuropathological mechanisms. Rev Neurosci. 2018;29(2):161-182. doi:10.1515/ revneuro-2017-0042 
117. Slota JA, Booth SA. MicroRNAs in neuroinflammation: implications in disease pathogenesis, biomarker discovery and therapeutic applications. Noncoding RNA. 2019;5(2):35. doi:10.3390/ ncrna5020035

118. Cui G-H, Guo H-D, Han L, et al. RVG-modified exosomes derived from mesenchymal stem cells rescue memory deficits by regulating inflammatory responses in a mouse model of Alzheimer's disease. Immun Ageing. 2019;16:10. doi:10.1186/ s12979-019-0150-2
119. Duan L, Limei X, Xiao X, et al. Exosome-mediated delivery of gene vectors for gene therapy. Nanoscale. 2021;13(3):1387-1397. doi:10.1039/d0nr07622h

120. Fowler CD. NeuroEVs:: Characterizing extracellular vesicles generated in the neural domain. $J$ Neurosci. 2019;39 (47):9262-9268. doi:10.1523/JNEUROSCI.0146-18.2019

\section{Publish your work in this journal}

Nature and Science of Sleep is an international, peer-reviewed, open access journal covering all aspects of sleep science and sleep medicine, including the neurophysiology and functions of sleep, the genetics of sleep, sleep and society, biological rhythms, dreaming, sleep disorders and therapy, and strategies to optimize healthy sleep.
The manuscript management system is completely online and includes a very quick and fair peer-review system, which is all easy to use. Visit http://www.dovepress.com/testimonials.php to read real quotes from published authors. 